\title{
Efficiency, accuracy, and stability issues in discrete-time simulations of single reed wind instruments
}

\author{
Federico Avanzini ${ }^{\mathrm{a})}$ \\ Dipartimento di Elettronica e Informatica, Università di Padova, Via Gradenigo 6/A, 35131 Padova, Italy \\ Davide Rocchesso ${ }^{\text {b) }}$ \\ Dipartimento di Informatica, Università di Verona, Strada Le Grazie 15, 37134 Verona, Italy
}

(Received 1 June 2000; accepted for publication 11 February 2002)

\begin{abstract}
A quantitative study of discrete-time simulations for a single reed physical model is presented. It is shown that when the continuous-time model is discretized, a delay-free path is generated in the computation. A general solution is proposed to this problem, that amounts to operating a geometrical transformation on the equations. The transformed equations are discretized using four different numerical methods. Stability properties of each method are assessed through analysis in the frequency domain. By comparing the discrete and continuous frequency responses, it is studied how the physical parameters are mapped by each method into the discrete-time domain. Time-domain simulations are developed by coupling the four digital reeds to an idealized bore model. Quantitative analysis of the simulations shows that the discrete-time systems produced by the four methods have significantly different behaviors, even when high sampling rates are used. As a result of this study, a general scheme for accurate and efficient time-domain simulations of the single reed model is proposed. (C) 2002 Acoustical Society of America. [DOI: 10.1121/1.1467674]
\end{abstract}

PACS numbers: 43.75.Pq [ADP]

\section{INTRODUCTION}

Numerical simulations are commonly used by musical acousticians for investigating experimentally the functioning of single reed wind instruments. ${ }^{1-3}$ A widely accepted approach divides the instrument into two main functional blocks, the acoustic bore (i.e., the resonator), and the reedmouthpiece system (sometimes referred to as the exciter). The resonator can be described through its reflection function (see Schumacher ${ }^{1}$ ). In this case, the pressure wave $p^{-}(t)$ reflected from the bore is obtained as time convolution of the reflection function with the incoming pressure wave $p^{+}(t)$. Another technique, waveguide modeling $\left(\mathrm{Smith}^{4}\right)$, divides the resonator into adjacent sections. In each section the wave propagation is simulated by means of two delay lines (left- and right-going). Terminations and junctions, as well as dissipation phenomena, are taken into account by inserting filtering elements in and between sections. Waveguide models are particularly accurate and efficient for simple cylindrical geometries, such as an idealized clarinet bore.

The airflow through the reed slit can be related to the reed opening and pressure through a nonlinear equation derived from the Bernoulli law. ${ }^{5}$ In the simplest approximation, the reed is assumed to move in phase with the driving pressure and is described only in terms of its stiffness. This is sometimes referred to as the quasistatic approximation. ${ }^{6}$ Using this approximation, the exciter can be described as a nonlinear map that relates flow and pressure at the bore entrance (see McIntyre et al. ${ }^{2}$ ). Despite its simplicity, such a quasistatic model is able to capture the basic nonlinear mechanisms that generate self-sustained oscillations in a

\footnotetext{
${ }^{a)}$ Electronic mail: avanzini@dei.unipd.it

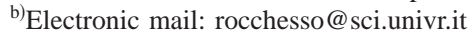

single reed instrument. Due to its compactness and the low number of parameters, this reed model can be efficiently used for sound synthesis purposes. ${ }^{4}$

A more accurate model takes into account other mechanical properties of the reed, namely its mass and the dissipation due to internal losses and air friction. In a first approximation, these elements are incorporated in the model by describing the reed as a damped second-order oscillator. ${ }^{1,5,7}$ This linear mechanical system is coupled with the nonlinear fluid dynamical equation. For clarity, in the following this model is referred to as the dynamic reed model.

In order to develop numerical simulations for the dynamic model, one has to overcome two main problems. First, the coupling in the equations typically generates a delay-free path in the computation. Due to the presence of a nonlinear equation, solving this loop is not trivial, unless one resorts to iterative solvers. Second, a numerical technique has to be chosen that preserves with reasonable accuracy the main properties of the physical system.

Concerning the first problem, many authors (see Gazengel $e t a l .^{3}$ ) compute the discrete-time equations by inserting a fictitious delay in the delay-free path. However, the numerical error introduced in this way can lead to instability, especially at low sampling rates. Anderson and Spong ${ }^{8}$ have proved analytically that the insertion of a delay element in a dynamical system deteriorates its stability properties. The second problem, i.e., the choice of an accurate discretization technique, is often neglected in the literature, and the equations are usually discretized using simple methods (such as the Euler method or the impulse invariant method), that introduce noticeable numerical artifacts in the simulations.

In this paper, an accurate and efficient discretization scheme for the dynamic reed model is developed. The delayfree loop in the computational scheme is solved by means of 
TABLE I. Variables and constants in the reed model.

\begin{tabular}{|c|c|c|}
\hline Quantity & Symbol & Value \\
\hline Sampling period & $T_{s}$ & $(\mathrm{~s})$ \\
\hline Sampling rate & $F_{s}$ & $\mathrm{~Hz})$ \\
\hline Frequency of the continuous-time system & $\omega_{c}$ & $(\mathrm{rad} / \mathrm{s})$ \\
\hline Frequency of the discretized systems & $\omega_{d}$ & $(\mathrm{rad} / \mathrm{s})$ \\
\hline Reed opening & $h$ & $(\mathrm{~m})$ \\
\hline Reed equil. opening & $h_{0}$ & $0.4 \cdot 10^{-3} \mathrm{~m}$ \\
\hline Reed displacement & $y_{r}$ & $(\mathrm{~m})$ \\
\hline Reed equil. displ. & $y_{0}$ & $(\mathrm{~m})$ \\
\hline Maximum allowed displ. & $y_{m}$ & $(\mathrm{~m})$ \\
\hline Reed mass/area & $\mu_{r}$ & $0.0231 \mathrm{~kg} / \mathrm{m}^{2}$ \\
\hline Effective flow surface & $S_{r}$ & $1.46 \cdot 10^{-4} \mathrm{~m}^{2}$ \\
\hline Reed resonance freq. & $\omega_{r}$ & $23250 \mathrm{rad} / \mathrm{s}$ \\
\hline Reed damping & $g_{r}$ & $3000 \mathrm{~s}^{-1}$ \\
\hline Amplitude parameter & $A$ & $0.0797 \mathrm{~m}^{3} /\left(\mathrm{N}^{2 / 3} \mathrm{~s}\right)$ \\
\hline Mouth pressure & $p_{m}$ & $(\mathrm{~Pa})$ \\
\hline Mouthpiece pressure & $p$ & $(\mathrm{~Pa})$ \\
\hline Pressure drop & $\Delta p$ & $(\mathrm{~Pa})$ \\
\hline Mouthpiece vol. flow & $u$ & $\left(\mathrm{~m}^{3} / \mathrm{s}\right)$ \\
\hline Flow through the slit & $u_{f}$ & $\left(\mathrm{~m}^{3} / \mathrm{s}\right)$ \\
\hline Wave impedance of the bore & $Z_{0}$ & $2290133 \mathrm{~kg} / \mathrm{m}^{4} \mathrm{~s}$ \\
\hline Speed of sound & $c$ & $347 \mathrm{~m} / \mathrm{s}$ \\
\hline Length of the bore & $L$ & $(\mathrm{~m})$ \\
\hline Bell cutoff freq. & $f_{\text {co }}$ & $1 \cdot 10^{3} \mathrm{~Hz}$ \\
\hline Pr. wave from the bore & $p^{-}$ & $(\mathrm{Pa})$ \\
\hline Pr. wave to the bore & $p^{+}$ & $(\mathrm{Pa})$ \\
\hline
\end{tabular}

the so-called $K$ method, recently proposed by Borin et al. ${ }^{9}$ The method operates a geometric transformation on the nonlinearity, in such a way that the delay-free path can be computed without introducing fictitious delays in the discretetime equations.

Given a general solution to noncomputability problems, four different numerical methods are used for discretizing the mechanical differential equations. The so-obtained "digital reeds" are analyzed in the frequency domain and compared to the continuous-time system. Then, the digital reeds are connected to a waveguide model of an ideal cylindrical bore, and the resulting systems are compared in the time domain through numerical simulations.

The choice of the discretization method is usually considered to be noncritical when simulations are run at high sampling rates. However, the results presented here show that this choice does affect the behavior of the numerical models noticeably, even at a sampling rate of several tens of $\mathrm{kHz}$ (e.g., $44.1 \mathrm{kHz}$ ). Among the considered techniques, the 1-step weighted sample method is found to be the most suitable choice for discretizing the dynamic reed model. This method accurately preserves the properties of the physical system, and its low computational costs make it suitable for real-time applications.

Section II describes the dynamic reed model. Numerical techniques are discussed in Sec. III. The frequency analysis presented in Sec. IV compares the digital reeds with the continuous-time system. Finally, Sec. V discusses results obtained from time-domain simulations.

Notation and symbols. Table I summarizes the symbols used throughout the paper. The parameter values are taken from Schumacher. ${ }^{1}$ Each time-varying variable $x$ is written as $x(t)$ and $x(n)$, respectively, in the continuous and discrete-

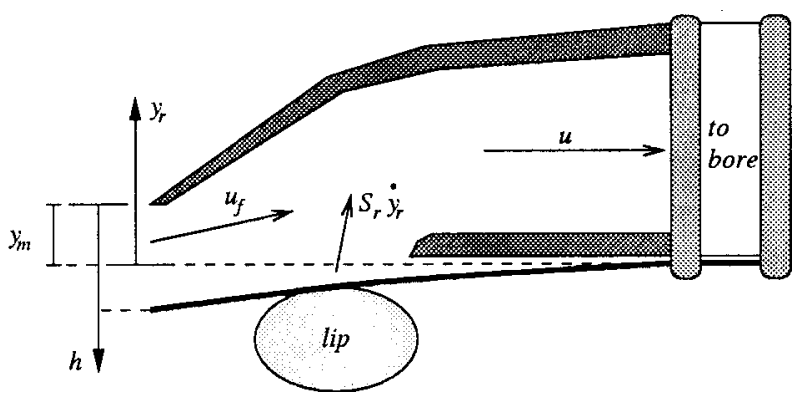

FIG. 1. Schematic representation of the reed-mouthpiece system.

time domains. The transforms of the continuous and discretetime signals are written, respectively, as $X(s)$ and $X(z)$.

\section{THE PHYSICAL MODEL}

\section{A. Exciter}

The dynamic model described below relies on the following assumptions: under normal playing conditions, oscillations occur mainly in the vertical direction, therefore a single degree of freedom (i.e., the reed tip vertical displacement) can be reasonably assumed; the reed resonances are well above the main frequency component of the driving pressure; therefore, only the effect of the first reed resonance needs to be modeled; the reed dimensions are small with respect to typical wavelengths in the resonator; thus, pressure can be thought of as constant along the reed internal surface. See Fig. 1.

Consequently, many authors ${ }^{1,5,7}$ approximate the reed as a harmonic oscillator, driven by the pressure drop $\Delta p=p_{m}$ $-p$ across the slit. When the reed beats (i.e., when it strikes the mouthpiece) an inelastic collision occurs:

$$
\begin{aligned}
& \ddot{y}_{r}(t)+g_{r} \dot{y}_{r}(t)+\omega_{r}^{2}\left[y_{r}(t)-y_{0}\right]=\Delta p(t) / \mu_{r}, \\
& \text { if } y_{r}<y_{m}, \\
& y_{r}(t)=y_{m} \text { and } \dot{y}_{r}(t)=0, \quad \text { if } y_{r} \geqslant y_{m} .
\end{aligned}
$$

The total airflow $u$ at the mouthpiece is the sum of two components. The first one is given by the flow $u_{f}$ through the slit. The second component is produced by the reed motion and depends on the reed velocity. This component is assumed to be $S_{r} \dot{y}_{r}$, where $S_{r}$ is the effective surface associated with the flow. Hence, the total flow $u$ is given by

$$
u(t)=u_{f}(t)+S_{r} \dot{y}_{r}(t) .
$$

The flow $u_{f}$ through the slit is related to the pressure drop $\Delta p$ and to the opening $h$ via the nonlinear equation

$$
\Delta p=F\left(u_{f}, h\right):=A^{-\alpha} \operatorname{sgn}\left(u_{f}\right)\left|u_{f}\right|^{\alpha} / h^{2} .
$$

With the value $\alpha=2$, Eq. (3) is the Bernoulli equation for an ideal fluid in the static regime. Using experiments on real instruments, Backus ${ }^{5}$ found empirically a value for $\alpha$ different from the theoretical one, namely $\alpha=3 / 2$. Backus suggested that this discrepancy could be due to the particular shape of the slit. More recently, Hirschberg et al. ${ }^{10}$ questioned the validity of Backus' experiments. These authors developed a flow model that uses the standard Bernoulli equation (with $\alpha=2$ ). 
In order to account for air inertance the term $M_{e} \dot{u}_{f}$ must be added to the right-hand side of Eq. (3) $\left(M_{e}\right.$ being the effective mass through the slit). The inclusion of this term complicates the model, since the computation of $\dot{u}_{f}$ is required. According to many authors, ${ }^{2,3,11}$ the effect of $M_{e}$ is generally small and this additional term can be neglected.

In summary, the dynamic reed model adopted in this work is fully described by the set of equations (1), (2), (3).

\section{B. Resonator}

The acoustical bore can be described by means of pressure waves $p^{ \pm}$, which by definition are related to pressure and airflow via the equations $p=p^{+}+p^{-}$and $u=\left(p^{+}\right.$ $\left.-p^{-}\right) / Z_{0}$. If cylindrical geometry is assumed and boundary losses are neglected, then the pressure wave $p^{+}$coming from the mouthpiece propagates freely with speed $c$ until it reaches the open end, terminated by the bell. The bell acts as a low-pass filter, reflecting low-frequency components inside the bore and radiating high-frequency components. Typical values for the cutoff frequency $f_{\text {co }}$ of the bell are between 1 and $1.5 \mathrm{kHz}$. The pressure wave $p^{-}$reflected from the bore to the mouthpiece is thus given by

$$
P^{-}(s)=-R_{c}(s) \exp (-s \quad 2 L / c) P^{+}(s) .
$$

$R_{c}(s)$ is the low-pass transfer function of the bell. The term $\exp (-s 2 L / c)$ accounts for the delay $2 L / c$ in the trip along the bore.

The waveguide model simulates the propagation with two delay lines. The length $m_{L}$ of each line is chosen in such a way that $m_{L} c T_{s}=L$. In the discrete domain the delay term $\exp (-s 2 L / c)$ is therefore replaced by $z^{-2 m_{L}}$. The low-pass bell filter is designed with standard techniques: ${ }^{13}$ first an ana$\log$ filter is designed using a fourth-order Butterworth realization; then, a digital equivalent $R_{d}(z)$ is obtained with usual methods from digital signal processing, such as the bilinear transformation

$$
R_{d}(z)=R_{c}\left(2 F_{s} \frac{1-z^{-1}}{1+z^{-1}}\right) .
$$

Summarizing, the digital bore model takes the incoming pressure wave $p^{+}$from the exciter, and reflects it as an outgoing pressure wave $p^{-}$given by

$$
P^{-}(z)=-R_{d}(z) z^{-2 m_{L}} P^{+}(z) .
$$

\section{NUMERICAL TECHNIQUES}

The waveguide techniques outlined in Sec. II B provide a model for the resonator in the discrete time-space domain. This section discusses the discrete-time approximation of the dynamic reed model. Equations (1), (2), and (3) generate the system

$$
\begin{aligned}
& \dot{\mathbf{w}}(t)=\mathbf{A} \mathbf{w}(t)+\mathbf{B u}(t)+\mathbf{c} \Delta p(t), \\
& \mathbf{x}(t)=\mathbf{D} \mathbf{w}(t)+\mathbf{E u}(t)+\mathbf{f} \Delta p(t), \\
& \Delta p(t)=F(\mathbf{x}(t)),
\end{aligned}
$$

where

$$
\mathbf{w}=\left[\begin{array}{c}
h \\
h
\end{array}\right], \quad \mathbf{u}=\left[\begin{array}{c}
h_{0} \\
p_{m} \\
p^{-}
\end{array}\right], \quad \mathbf{x}=\left[\begin{array}{c}
u_{f} \\
h
\end{array}\right],
$$

and

$\mathbf{A}=\left[\begin{array}{cc}0 & 1 \\ -\omega_{r}^{2} & -g_{r}\end{array}\right], \quad \mathbf{B}=\left[\begin{array}{ccc}0 & 0 & 0 \\ \omega_{r}^{2} & 0 & 0\end{array}\right], \quad \mathbf{c}=\left[\begin{array}{c}0 \\ -1 / \mu_{r}\end{array}\right]$,
$\mathbf{D}=\left[\begin{array}{cc}0 & -S_{r} \\ 1 & 0\end{array}\right], \quad \mathbf{E}=\left[\begin{array}{ccc}0 & 1 / Z_{0} & -2 / Z_{0} \\ 0 & 0 & 0\end{array}\right], \quad \mathbf{f}=\left[\begin{array}{c}-1 / Z_{0} \\ 0\end{array}\right]$.

The beating condition in Eq. (1) turns into

$$
\mathbf{w}=\mathbf{0} \text {, if } h \leqslant 0 \text {. }
$$

\section{A. Solving the delay-free loop}

When the first equation in system (7) is discretized, the structure of the resulting difference equation is found to be

$$
\mathbf{w}(n)=\widetilde{\mathbf{w}}(n)+\overline{\mathbf{c}} \Delta p(n) .
$$

The vector $\widetilde{\mathbf{w}}(n)$ is a linear combination of all the terms that are computable at time $n$ [namely, $\mathbf{u}(n)$ and past values of $\mathbf{w}$, $\mathbf{u}$, and $\Delta p$ ], while the vector $\overline{\mathbf{c}}$ weights the dependence of $\mathbf{w}$ on $\Delta p(n)$. Explicit expressions for both $\widetilde{\mathbf{w}}(n)$ and $\overline{\mathbf{c}}$ depend on the numerical method actually used. The remaining equations in system (7) can thus be written as

$$
\begin{aligned}
& \mathbf{x}(n)=\widetilde{\mathbf{x}}(n)+\mathbf{k} \Delta p(n), \\
& \Delta p(n)=F(\widetilde{\mathbf{x}}(n)+\mathbf{k} \Delta p(n)),
\end{aligned}
$$

where $\mathbf{k}=(\mathbf{D} \overline{\mathbf{c}}+\mathbf{f})$. The vector $\widetilde{\mathbf{x}}(n)=\mathbf{D} \widetilde{\mathbf{w}}(n)+\mathbf{E u}(n)$ does not depend on $\Delta p(n)$ and is therefore computable at each step. In the second equation (13), the term $\Delta p(n)$ depends implicitly on itself. In order to compute this equation, the $K$ method is used. ${ }^{9}$ This method uses the implicit mapping theorem to operate a geometric transformation on the nonlinear function $F$, and turns the second equation (13) into an explicit dependence

$$
\begin{aligned}
\Delta p(n) & =F(\widetilde{\mathbf{x}}(n)+\mathbf{k} \Delta p(n)), \\
K & \text { method } \\
& \mapsto \Delta p(n)=\bar{F}(\widetilde{\mathbf{x}}(n)) .
\end{aligned}
$$

Therefore, at each time-step $n$ the vector $\widetilde{\mathbf{x}}(n)$ is computed first, then $\Delta p(n)$ is obtained through the new nonlinear relation $\bar{F}$. Although $\bar{F}(\widetilde{\mathbf{x}})$ is not available in closed form in most cases, an implementation can still be obtained without resorting to iterative solvers, by storing the multivariable function $\bar{F}$ as a set of precomputed tables. ${ }^{14}$

\section{B. Discretizing the equations}

The $\mathrm{K}$ method provides a robust and general means to compute the difference equations (13) accurately. Given such a method, different discretization techniques for system (7) can be compared.

Typical choices in the literature are the Euler method and the impulse invariant method. However, both these techniques introduce artifacts in the numerical systems. Gazengel et $a .^{3}{ }^{3}$ discuss the use of a fourth-order Runge-Kutta solver. 
This method, although very accurate when high sampling rates are used, turns out to be unstable at low $F_{s}$. Moreover, it has high computational costs, since four evaluations of the nonlinear function $F(\mathbf{x}(n))$ are needed at each time step. Van Walstijn $^{15}$ uses a hybrid backward-centered scheme that approximates the first derivative with the backward Euler rule and the second derivative with a centered difference. One advantage of this approach is that the vectors $\overline{\mathbf{c}}$ and $\mathbf{k}$ in Eqs. (12) and (13) are both zero; therefore, no delay-free paths are created in the discrete-time equations. However, at each time step $n$ the Newton-Raphson method is used for computing iteratively the flow $u(n)$, and nine iterations are typically required.

The following techniques are used in the rest of this paper.

1- and 2-step Adams-Moulton methods (AM1, 2 from now on). These are linear multistep methods, whose stability and accuracy properties are known from the numerical analysis literature. ${ }^{16}$

1- and 2-step weighted sample methods (WS1, 2 from now on). These have been introduced recently by Wan and Schneider. ${ }^{17}$ They are designed for generic linear systems, and are based on a polynomial interpolation of the input.

Higher-order methods are not used for two main reasons: (1) stability properties tend to deteriorate with increasing order, and (2) the computational costs become higher.

As Schneider et al. ${ }^{18}$ have pointed out, AM methods can be easily seen as $s$-to- $z$ mappings in the complex plane

$$
\begin{aligned}
& (\mathrm{AM} 1) s=2 F_{s} \frac{1-z^{-1}}{1+z^{-1}}, \\
& (\mathrm{AM} 2) s=12 F_{s} \frac{1-z^{-1}}{5+8 z^{-1}-z^{-2}} .
\end{aligned}
$$

Note that the mapping (15a) associated to the AM1 method is the bilinear transformation.

Applying the AM methods to the first equation in system (7) amounts to Laplace-transforming it and substituting each occurrence of $s$ with the corresponding mapping (15a) or (15b). Therefore, the differential equation is turned into a second-order and a fourth-order difference equation, by the AM1 and the AM2 methods, respectively. If system (7) is time-invariant, then the AM methods provide a timeinvariant discrete system. If some of the reed parameters are varied over time, then the discrete system coefficients need to be updated at a suitable control rate.

Wan and Schneider ${ }^{17}$ have shown that the $k$-step WS method turns the first equation in system (7) into the difference equation

$$
\begin{aligned}
\mathbf{w}(n)= & \boldsymbol{\Phi}\left(T_{s}\right) \mathbf{w}(n-1)+\mathcal{W}_{\mathbf{u}} \mathbf{S}_{\mathbf{u}}{ }^{(k)}\left[\begin{array}{c}
\mathbf{u}(n) \\
\vdots \\
\mathbf{u}(n-k)
\end{array}\right] \\
& +\mathcal{W}_{\Delta p} \mathbf{S}_{\Delta p}^{(k)}\left[\begin{array}{c}
\Delta p(n) \\
\vdots \\
\Delta p(n-k)
\end{array}\right],
\end{aligned}
$$

where $k=1,2$. Therefore, the differential equation is turned into a second-order and a third-order difference equation, respectively, by the WS1 and the WS2 methods.

Details about the computation of the matrices can be found elsewhere. ${ }^{17,19}$ The matrices $\mathbf{S}_{\mathbf{u}}^{(k)}$ and $\mathbf{S}_{\Delta p}^{(k)}$ are interpolation matrices that depend only on the order $k$ of the method, while $\boldsymbol{\Phi}\left(T_{s}\right)$ is the exponential matrix defined from $\boldsymbol{\Phi}(t)=\exp (\mathbf{A} t)$. Computation of $\mathcal{W}_{\mathbf{u}}, \mathcal{W}_{\Delta p}$ involves calculation of the $k+1$ integrals $\int_{0}^{T} \boldsymbol{\Phi}\left(T_{s}-t\right) \cdot t^{l} d t$ (for $l=1 \cdots k$ $+1)$. Therefore, computation of the coefficients in the difference equation (16) requires computation of transcendental functions.

If system (7) is time-invariant, then computation of matrices $\boldsymbol{\Phi}\left(T_{s}\right), \mathcal{W}_{\mathbf{u}}$, and $\mathcal{W}_{\Delta p}$ can be performed off-line, while these matrices need to be updated at control rate when time-varying control parameters are used. In this latter case the WS methods have higher computational costs than the AM methods, and this is a potential drawback for real-time applications. However, in the case of low-order methods $(k=1,2)$, only a small number of coefficients needs to be updated. Moreover, Wan and Schneider ${ }^{17}$ show that the computational costs can be lowered using ad hoc techniques (e.g., the columns of $\mathcal{W}$ can be computed iteratively).

\section{Properties of the methods}

Stability properties of an AM method are summarized by the shape of its region of absolute stability $\mathcal{R}_{A}$ (see Lambert). ${ }^{16}$ If the continuous-time eigenvalues $\lambda_{l}(l$ $=1, \ldots, N)$ of a stable, $N$-dimensional continuous system lie inside $\mathcal{R}_{A}$, then the discretized system is stable. As far as the bilinear transformation is concerned, it is known that $\mathcal{R}_{A}$ is the whole left-half $s$-plane. Therefore, continuous-time eigenvalues $\lambda_{l}$ with $\operatorname{Re}\left(\lambda_{l}\right)<0$ are mapped into discrete-time eigenvalues $p_{l}$ with $\left|p_{l}\right|<1$, and stability is preserved at any sampling rate. The AM2 method has worse stability properties, since its region of absolute stability is the finite subset of the left-half $s$-plane shown in Fig. 2. This means that stability is preserved only at high sampling rates, so that the eigenvalues of the continuous system lie inside $\mathcal{R}_{A}$.

For the k-step WS methods, Wan and Schneider have shown that the discrete-time eigenvalues are the roots of the characteristic polynomial $p(z)=z^{k-1}\left|z \mathbf{I}-\boldsymbol{\Phi}\left(T_{s}\right)\right|$. Therefore, continuous-time eigenvalues $\lambda_{l}$ map into discrete-time eigenvalues $p_{l}$ through the relation

$$
p_{l}=\exp \left(\lambda_{l} T_{s}\right) \text {. }
$$

If $\operatorname{Re}\left(\lambda_{l}\right)<0$, then $\left|p_{l}\right|<1$; therefore, stability is preserved at any sampling rate. Note that the same relation (17) between discrete- and continuous-time eigenvalues holds for the impulse invariant method. Indeed, it can be verified that the WS method with $k=0$ is completely equivalent to the impulse invariant method.

Concerning accuracy, it is a general result that the k-step AM method has order $k+1$. This means that the methods provide a global truncation error in time which has order $T_{s}^{k+1}$. For the WS methods, Wan and Schneider have given experimental results showing that a k-step method has order $k+1$, the same as the corresponding AM method. 


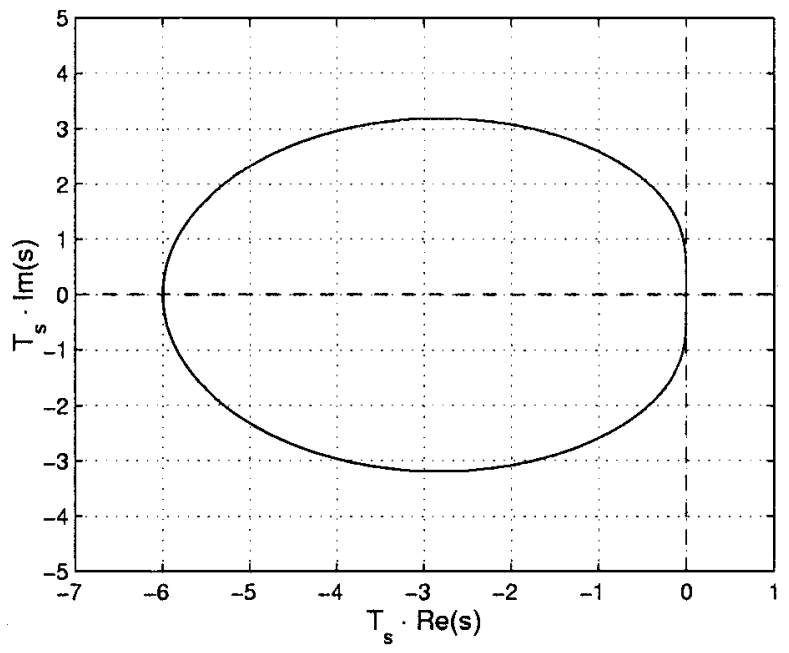

FIG. 2. Region of absolute stability $\mathcal{R}_{A}$ for the AM2 method.

\section{THE DIGITAL REEDS}

This section is devoted to frequency analysis of the digital reeds obtained using AM and WS methods. Such analysis permits comparison of the different discretization techniques, by studying how the physical parameters are mapped into the discrete-time domain.

Consider the transfer function $H_{c}(s)$ of the relative displacement $y_{r}-y_{0}$ versus the pressure drop $\Delta p$. From Eq. (1), this is seen to be the harmonic oscillator

$$
H_{c}(s)=\frac{1}{\mu_{r}} \frac{1}{s^{2}+g_{r} s+\omega_{r}^{2}} .
$$

The frequency response is given by $H_{c}\left(j \omega_{c}\right)$. A meaningful comparison between the numerical methods amounts to analyzing how they preserve this frequency response in the discrete-time domain. The study is performed for various $F_{s}$. Typical audio sampling rates $F_{s}=22.05 \mathrm{kHz}$ and $F_{s}=44.1 \mathrm{kHz}$ are taken as reference values. Following ideas developed by Gazengel et al., ${ }^{3}$ the analysis is focused on three physically meaningful parameters of the dynamic model: the resonance frequency $\omega_{r}$, the oscillator stiffness (defined as $\left|H_{c}(0)\right|^{-1}=\mu_{r} \omega_{r}^{2}$ ), and the damping coefficient $g_{r}$.

Typical values for $\omega_{r}$ lie in the high-frequency region, and this parameter is therefore considered to be noncritical in helping self-sustained oscillations. Indeed, self-sustained oscillations occur even when there is no resonance at all, as in the quasistatic approximation (see Sec. V B in the following). However, as pointed out by Thompson, ${ }^{12}$ the reed resonance has a role in adjusting pitch, loudness, and tone color, as well as helping transitions to high regimes of oscillation, such as the clarion register or the reed regime ("squeaks"). Stiffness characterizes the reed response in the low-frequency region and is therefore an important parameter, since the fundamental frequency of the oscillation always lies in this region. Concerning the damping coefficient, the relation $g_{r}=\omega_{r} / Q_{r}$ holds for the harmonic oscillator (18), where $Q_{r}=\omega_{r} /\left(\omega_{1}-\omega_{2}\right)$ is the quality factor and $\omega_{1,2}$ are the 3-dB cutoff frequencies. Therefore, $g_{r}=\omega_{1}-\omega_{2}$ for the continuous-time oscillator (18).
When using AM and WS methods, $H_{c}(s)$ is turned into a digital filter which is not a harmonic oscillator. Therefore, the parameters $\omega_{r}, \mu_{r}, g_{r}$ cannot be deduced from the coefficients of the discrete-time transfer functions. Instead, they are extrapolated from the shape of the discrete-time frequency responses. In particular, following Gazengel et al. ${ }^{3}$ the digital damping coefficient is defined in the following as $g_{d}=\omega_{d 1}-\omega_{d 2}$, where $\omega_{d 1, d 2}$ are the 3 - $\mathrm{dB}$ cutoff frequencies for the response of the discrete-time system.

\section{A. Adams-Moulton methods}

Using the AM methods, the digital transfer functions $H_{\mathrm{AM} 1}(z)$ and $H_{\mathrm{AM} 2}(z)$ are obtained by substitution of the corresponding $s$-to- $z$ mapping [(15a), (15b)] in $H_{c}(s)$. The corresponding frequency responses are given by evaluation at $z=\exp \left(j \omega_{d} / F_{s}\right)$. From Sec. IV, $H_{\mathrm{AM} 1}(z)$ and $H_{\mathrm{AM} 2}(z)$ are known to have order 2 and 4 , respectively.

The frequency responses $H_{\mathrm{AM} 1}$ and $H_{\mathrm{AM} 2}$ of the discretized systems are plotted in Fig. 3 for the cases $F_{s}=22.05 \mathrm{kHz}$ and $F_{s}=44.1 \mathrm{kHz}$. Responses obtained with the Euler method are also plotted as a term of comparison.

The Euler method is seen to provide poor accuracy even at $F_{s}=44.1 \mathrm{kHz}$. In particular, a noticeable numerical dissipation is introduced, so that the resonance is heavily attenuated. Results for AM methods are in good agreement with theoretical predictions. Both the magnitude and the phase responses of $H_{\mathrm{AM} 1}$ exhibit a known "frequency warping" phenomenon: the induced map between the continuous frequency $\omega_{c}$ and discrete frequency $\omega_{d}$ is (see Mitra) ${ }^{13}$ $\omega_{d}=2 F_{s} \arctan \left(\omega_{c} / 2 F_{s}\right)$. High frequencies are thereby compressed, and this phenomenon becomes more noticeable as the sampling rate decreases; at $F_{s}=22.05 \mathrm{kHz}$ the resonance $\omega_{r}$ of $H_{\mathrm{AM} 1}$ has shifted from the original value 23250 $\mathrm{rad} / \mathrm{s}$ to the value $21300 \mathrm{rad} / \mathrm{s}$ (i.e., from $3700 \mathrm{~Hz}$ to 3390 $\mathrm{Hz}$ ).

The AM2 method provides different results: there is no significant warping, but the magnitude of the resonance is amplified. The amplification is small at $F_{s}=44.1 \mathrm{kHz}$, but becomes unacceptable at $F_{s}=22.05 \mathrm{kHz}$ (the peak magnitude is $4.7 \cdot 10^{-5} \mathrm{~m} / \mathrm{Pa}$ ). This phenomenon is a direct consequence of stability properties. Indeed, it can be seen that, using the values listed in Table I, the method becomes unstable at $F_{s} \simeq 19 \mathrm{kHz}$. This explains the strong amplification and the phase distortion exhibited by $H_{\mathrm{AM} 2}$ at $F_{s}=22.05 \mathrm{kHz}$.

Both the methods preserve stiffness [the equalities $H_{\mathrm{AM} 1}(0)=H_{\mathrm{AM} 2}(0)=H_{c}(0)$ hold]. Finally, qualitative analysis shows that both methods lead to a digital damping $g_{d}=\omega_{d 1}-\omega_{d 2}$ that is smaller than the physical one, and decreases with decreasing $F_{s}$. For $H_{\mathrm{AM} 1}$ this is a consequence of frequency warping, which causes the resonance bandwidth to reduce, and the quality factor to increase consequently. For $H_{\mathrm{AM} 2}$ this is due to the resonance amplification rather than to warping.

\section{B. Weighted sample methods}

The 1- and 2-step WS methods do not define a $s$-to- $z$ mapping; therefore, the discrete-time transfer functions 

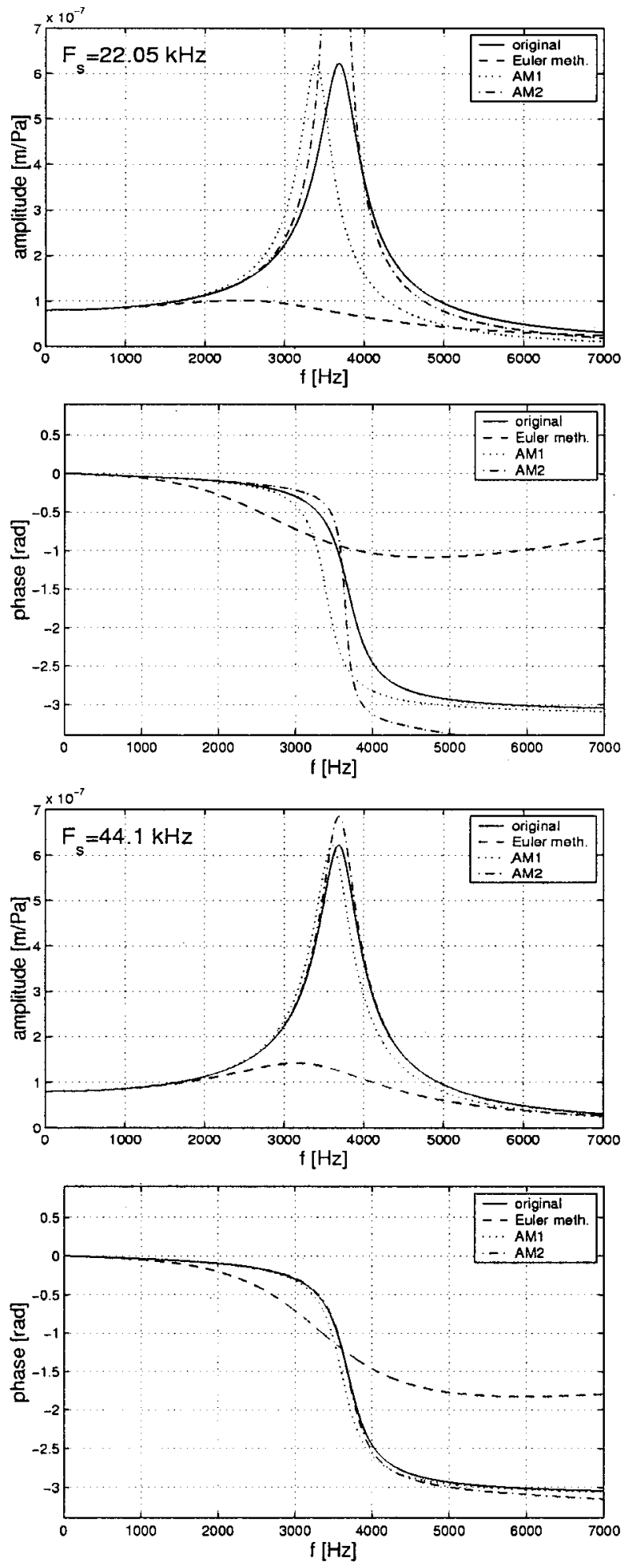

FIG. 3. Frequency responses from AM methods, with $F_{s}$ $=22.05,44.1 \mathrm{kHz} . H_{c}$ (solid line), Euler method discretization (dashed), $H_{\mathrm{AM} 1}$ (dotted), $H_{\mathrm{AM} 2}$ (dot-dashed).

$H_{\mathrm{WS} 1}(z)$ and $H_{\mathrm{WS} 2}(z)$ are not obtained by substitution. Instead, they are computed directly from the general equation (16). From Sec. IV, it can be seen that $H_{\mathrm{Ws} 1}(z)$ and $H_{\mathrm{Ws} 2}(z)$ have order 2 and 3, respectively.

Results are summarized in Fig. 4. Responses obtained with the Euler method are plotted as a term of comparison.

The discrete-time responses $H_{\mathrm{wS} 1}$ and $H_{\mathrm{wS} 2}$ show ex- cellent agreement with $H_{c}$, even at low sampling rates. Both methods preserve the resonance $\omega_{r}$ without introducing warping. Stiffness is preserved as well. Numerical dissipation is introduced, which is more significant for the 1-step method. This can be noticed by observing that the digital amplitude responses lie below the continuous one. Due to this dissipation, the digital damping coefficient $g_{d}$ is larger than the physical one and increases with decreasing $F_{s}$, for both $H_{\mathrm{ws} 1}(z)$ and $H_{\mathrm{ws} 2}(z)$. Phase responses are well preserved by both methods.

Summarizing, the frequency analysis developed in this section has shown that the WS methods better approximate the reed frequency response than AM methods. It would appear that the WS methods are preferable. However, this conjecture is not confirmed from the time-domain analysis developed in the next section.

\section{TIME-DOMAIN SIMULATIONS}

In order to obtain time-domain simulations, each of the four digital reeds is coupled to the same resonator (the waveguide cylindrical bore described in Sec. II) to form a complete instrument. Comparisons of simulations lead to two main results, which are not evident from the frequency analysis of Sec. IV.

(i) the systems can behave differently even at high sampling rates, where the reed frequency responses are indistinguishable;

(ii) both the 1-step methods approximate the continuous system accurately, while the 2-step methods exhibit artifacts.

\section{A. Threshold pressure}

A first simulation study concerns the threshold pressure $p_{t}$, defined as the value of mouth pressure above which stable oscillations take place. A rough estimate for the threshold pressure, $p_{t} \simeq h_{0} \mu_{r} \omega_{r}^{2} / 3$, can be derived using the quasistatic approximation (see Hirschberg et al. ${ }^{6}$ and Sec. V B). With the values listed in Table I, the "quasistatic estimate" is $p_{t} \simeq 1664 \mathrm{~Pa}$. However, as observed by Keefe, ${ }^{20}$ this value underestimates the true $p_{t}$.

In this section such a quasistatic estimate is compared with experimental results from simulations. First, a "dynamic estimate" is found by running simulations at very high sampling rates (up to $500 \mathrm{kHz}$ ). For such sampling rates, all the systems are found to have the same threshold pressure, $p_{t}=1802 \mathrm{~Pa}$. This is therefore assumed to be the "true" value. Then, simulations are run at lower sampling rates: the measured $p_{t}$ are given in Table II, from which some remarks can be made.

(i) For all the digital reeds, $p_{t}$ converges to the dynamic estimate $1802 \mathrm{~Pa}$ as the sampling rate is increased. The convergence of AM2 is not evident from Table II, since it occurs at $F_{s}>200 \mathrm{kHz}$.

(ii) The $p_{t}$ estimates obtained from both the 1-step methods exhibit robustness with respect to the sampling rate. At $F_{s}=30 \mathrm{kHz}$, the deviation of $p_{t}$ from the true value is less than $1 \%$ for both AM1 and WS1. 

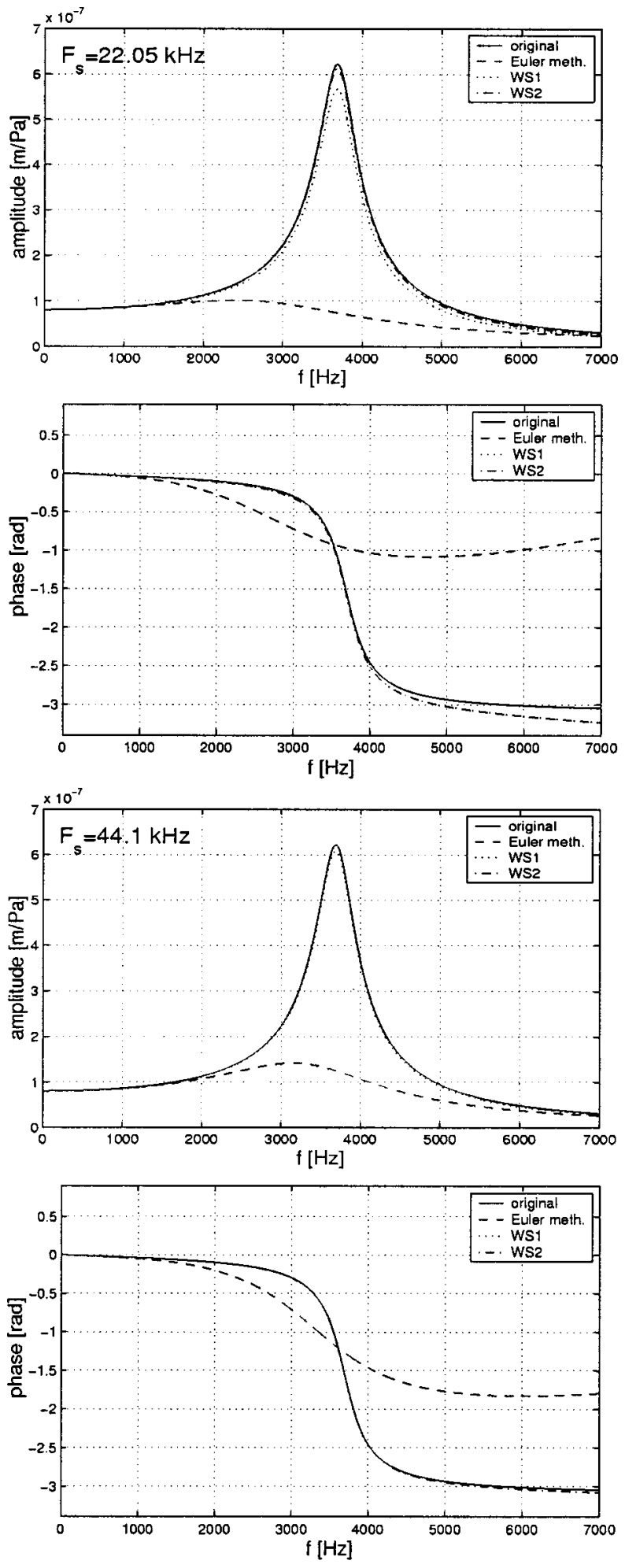

FIG. 4. Frequency responses from WS methods, with $F_{s}$ $=22.05,44.1 \mathrm{kHz} . H_{c}$ (solid line), Euler method discretization (dashed), $H_{\mathrm{wS} 1}$ (dotted), $H_{\mathrm{ws} 2}$ (dot-dashed).

(iii) The 2-step methods are less robust: even at high sampling frequencies, the threshold pressures for the corresponding systems are far from the true value. In particular, simulations with AM2 hardly reach steadystate oscillations for $F_{s}<35 \mathrm{kHz}$. For this reason the AM2 column in Table II provides results only for $F_{s}>50 \mathrm{kHz}$.
Consider the frequency response $H_{\mathrm{WS} 2}$ at $F_{s}$ $=44.1 \mathrm{kHz}$ : from Fig. 4 , this is seen to be indistinguishable from the original one. However, the estimated $p_{t}$ is still noticeably higher than the true value. An analogous remark holds for AM2 with $F_{s}=100 \mathrm{kHz}$. Therefore, the 2-step methods exhibit poor accuracy even when the reed response is well approximated.

These results show that the discrete-time frequency response does not provide sufficient information on the digital reed when this is coupled with the nonlinearity $F\left(u_{f}, h\right)$ and with the bore. Due to the nonlinearity, the whole system exhibits sensitive dependence on small deviations in the frequency response.

\section{B. Dynamic versus quasistatic}

In this section, the dynamic model is compared with the quasistatic reed approximation. This approximation provides a simplified description of reed motion by exploiting the following: the reed resonance is noticeably higher than the playing frequency of any playable note on the clarinet; therefore, the spectrum of $\Delta p$ is confined to the low-frequency region, where it is reasonable to assume a flat reed response (see Figs. 3, 4). The response $H_{c}\left(j \omega_{c}\right)$ is therefore approximated by the zero-frequency response $H_{c}(0)=1 /\left(\mu_{r} \omega_{r}^{2}\right)$, and in this approximation the reed moves in phase with the pressure drop according to the relation

$$
\left[y_{r}(t)-y_{0}\right]=H_{c}(0) \Delta p(t) .
$$

Substituting Eq. (19) in Eq. (3), and recalling that $h=y_{m}$ $-y_{r}$, a few calculation steps yield

$$
u_{f}=A\left(h_{0}-\Delta p /\left(\mu_{r} \omega_{r}^{3}\right)\right)^{4 / 3} \cdot \operatorname{sgn}(\Delta p)|\Delta p|^{2 / 3} .
$$

The properties of this memoryless model have been studied by many authors. ${ }^{2,4,6,21}$

A first comparison between the quasistatic and the dynamic models amounts to plotting the corresponding phase diagrams for the steady-state signals $u_{f}$ and $\Delta p$. Figure 5 shows an example of such phase diagram, obtained from numerical simulations with the WS1 method with a mouth pressure $p_{m}=2265 \mathrm{~Pa}$. This value is the maximum value for nonbeating conditions.

The phase diagram for the quasistatic model is simply the plot of Eq. (20), while the dynamic model exhibits a more interesting behavior: $u_{f}$ and $\Delta p$ move along a hysteretic path. This is due to the presence of memory in the equations: when the reed dynamics is taken into account, then Eqs. (19), (20) do not hold, and $h$ and $u_{f}$ depend on $\Delta p$ together with its derivatives. In other words, the attractor in the dynamic reed model is not a curve in the plane, but instead a closed orbit in a higher-dimensional phase space.

Another important difference in the behavior of quasistatic and dynamic simulations concerns transitions to high regimes of oscillation. As Thompson ${ }^{12}$ and Wilson and Beavers ${ }^{7}$ have pointed out, both $\omega_{r}$ and $g_{r}$ play a key role in helping transition to the second register (clarion register). Experiments with artificial lips and real clarinets have shown that the clarion register can be produced without opening the register hole, if the reed resonance matches a low harmonic of the playing frequency and the damping is small enough. 
TABLE II. Measured threshold pressures from time-domain simulations.

\begin{tabular}{|c|c|c|c|c|c|}
\hline \multirow[b]{2}{*}{$F_{s}(\mathrm{kHz})$} & \multicolumn{5}{|c|}{$p_{t}(\mathrm{~Pa})$} \\
\hline & Q. Static & AM1 & WS1 & $\mathrm{AM} 2$ & WS2 \\
\hline 20 & 1664 & 1816 & 1761 & $\ldots$ & 3346 \\
\hline 25 & 1664 & 1808 & 1774 & $\cdots$ & 2842 \\
\hline 30 & 1664 & 1807 & 1784 & $\cdots$ & 2554 \\
\hline 35 & 1664 & 1807.5 & 1790 & $\cdots$ & 2365 \\
\hline 40 & 1664 & 1807.5 & 1795 & $\cdots$ & 2233 \\
\hline 45 & 1664 & 1804 & 1796 & $\cdots$ & 2136 \\
\hline 50 & 1664 & 1804.5 & 1797 & 3781 & 2063 \\
\hline 55 & 1664 & 1805 & 1798 & 3516 & 2008 \\
\hline 60 & 1664 & 1805.5 & 1799 & 3278 & 1960 \\
\hline 65 & 1664 & 1806 & 1799.5 & 3148 & 1932 \\
\hline 70 & 1664 & 1803.5 & 1800 & 3026 & 1906 \\
\hline 75 & 1664 & 1804 & 1800.5 & 2908 & 1881 \\
\hline 80 & 1664 & 1804.5 & 1801 & 2841 & 1865 \\
\hline 85 & 1664 & 1805 & 1801.5 & 2887 & 1848 \\
\hline 90 & 1664 & 1803 & 1802 & 2737 & 1832 \\
\hline 95 & 1664 & 1803.5 & 1802 & 2692 & 1816 \\
\hline 100 & 1664 & 1803.5 & 1802 & 2643 & 1802 \\
\hline
\end{tabular}

Moreover, extremely low damping causes the reed regime ("squeaks") to be produced. From a musical standpoint, squeaks are often explained as a consequence of insufficient breathing, while the fundamental register comes in as mouth pressure is increased.

All these effects are reproduced using the dynamic model, while the quasistatic model does not provide control over such effects. Figure 6(a) shows examples of transitions from numerical simulations with the WS1 method. The clarion register is obtained by matching $\omega_{r}$ to the seventh harmonic of the playing frequency and lowering $g_{r}$ to 1400 $\mathrm{s}^{-1}$. In Fig. 6(b) the reed regime is achieved by giving $g_{r}$ a value as low as $300 \mathrm{~s}^{-1}$. Squeaks are more easily obtained in simulations by driving the reed with low blowing pressures.

\section{CONCLUSIONS}

In this paper, a simulation scheme for the single reed system was proposed, resulting in two conclusions. First, analysis of the discrete-time frequency responses was performed in order to study how theoretical properties of the numerical methods, such as stability, accuracy, and fre-

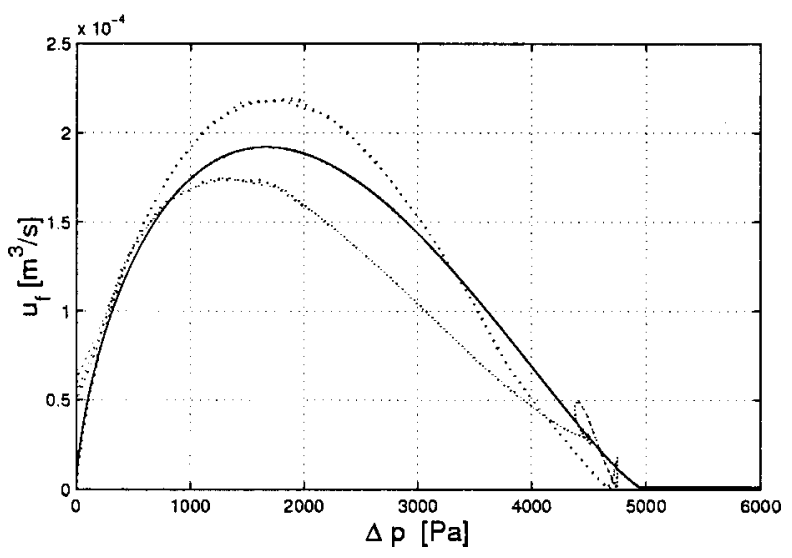

FIG. 5. Phase diagrams for $u_{f}$ vs $\Delta p$. Quasistatic model (solid line), and dynamic model (dotted line) discretized with the WS1 method $\left(F_{s}=44.1 \mathrm{kHz}, p_{m}=2265 \mathrm{~Pa}\right)$. quency warping, affect the reed behavior in the digital domain. It has been shown that 1-step methods, such as the bilinear transformation or the 1-step weighted sample method, can approximate the system with good accuracy while keeping the computational costs low. Second, timedomain simulations were obtained by coupling the single reed to a simple bore. These have shown that the analysis of the reed frequency responses do not provide enough information on the properties of the whole system due to nonlinear coupling in the equations. Indeed, the discrete-time models have significantly different behaviors even when the
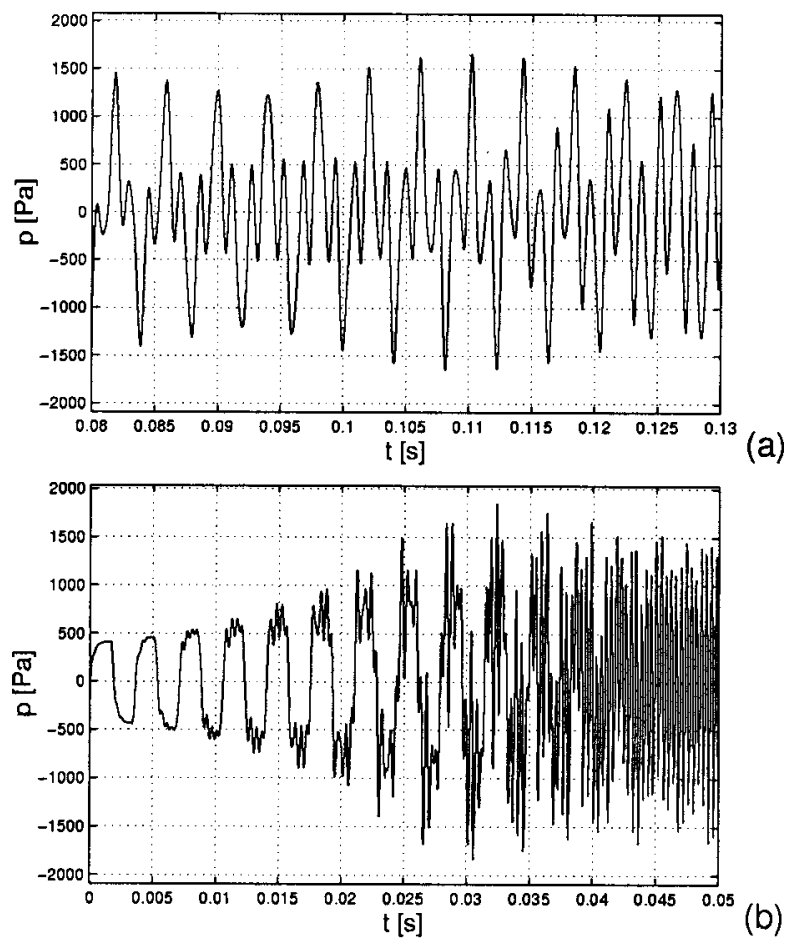

FIG. 6. Transitions to high regimes of oscillation (WS1 method, $\left.F_{s}=44.1 \mathrm{kHz}\right) ; \quad$ (a) clarion register $\left(\omega_{r}=2 \pi \cdot 2020 \mathrm{rad} / \mathrm{s}, g_{r}=1400 \mathrm{~s}^{-1}\right.$, $\left.p_{m}=1800 \mathrm{~Pa}\right) ; \quad$ (b) reed regime $\left(\omega_{r}=2 \pi \cdot 3150 \mathrm{rad} / \mathrm{s}, g_{r}=300 \mathrm{~s}^{-1}\right.$, $\left.p_{m}=1900 \mathrm{~Pa}\right)$. 
frequency responses of the digital reeds are almost indistinguishable. Furthermore, the 2-step methods introduce artifacts in the digital domain while 1-step methods better approximate the original system.

These two results show that the 1-step weighted sample method provides the most accurate reed simulations. Due to its low computational costs, it can be used as an efficient tool for sound synthesis purposes, in combination with the $\mathrm{K}$ method. Moreover, low sampling rates can be used without introducing instability or serious artifacts. This is important in emerging applications such as structured audio coding, ${ }^{22}$ where instrument models are encoded and transmitted without precise knowledge of the computational power of the decoder.

The present study has focused on the accurate simulation of the instrument exciter. Good sound quality from numerical simulations can only be achieved if also the resonator is modeled accurately (dissipation and dispersion, toneholes, radiation from the bell, etc.) ${ }^{23}$ therefore, the bore model presented in Sec. II B is far too poor to provide satisfactory sound quality. Nonetheless, sound examples obtained from numerical simulations ${ }^{24}$ show that reed physical parameters allow effective and realistic control over the digital instrument.

\section{ACKNOWLEDGMENTS}

The authors would like to thank Gianpaolo Borin and Giovanni De Poli for constant support throughout this research, and Mark Kahrs for providing a number of helpful remarks and comments on a preliminary version of the manuscript.

${ }^{1}$ R. T. Schumacher, "Ab initio calculations of the oscillations of a clarinet," Acustica 48(2), 71-85 (1981).

${ }^{2}$ M. E. McIntyre, R. T. Schumacher, and J. Woodhouse, "On the oscillations of musical instruments," J. Acoust. Soc. Am. 74, 1325-1345 (1983).

${ }^{3}$ B. Gazengel, J. Gilbert, and N. Amir, "Time domain simulation of single reed wind instrument. From the measured impedance to the synthesis signal. Where are the traps?," Acta Acust. 3, 445-472 (1995).

${ }^{4}$ J. O. Smith III, "Principles of Digital Waveguide Models of Musical Instruments," in Applications of DSP to Audio and Acoustics, edited by M. Kahrs and K. Brandenburg (Kluwer Academic, Dordrecht, 1998), Chap. 10, pp. 417-466.
${ }^{5}$ J. Backus, "Small-vibration theory of the clarinet," J. Acoust. Soc. Am. 35, 305-313 (1963).

${ }^{6}$ A. Hirschberg, X. Pelorson, and J. Gilbert, "Aeroacoustics of musical instruments," Meccanica 31, 131-141 (1996).

${ }^{7}$ T. A. Wilson, and G. S. Beavers, "Operating modes of the clarinet," J. Acoust. Soc. Am. 56, 653-658 (1974).

${ }^{8}$ R. J. Anderson and M. W. Spong, "Bilateral control of teleoperators with time delay," IEEE Trans. Autom. Control 34, 494-501 (1989).

${ }^{9}$ G. Borin, G. De Poli, and D. Rocchesso, "Elimination of delay-free loops in discrete-time models of nonlinear acoustic systems," IEEE Trans. Speech Audio Process. 8(5), 597-606 (2000).

${ }^{10}$ A. Hirschberg, R. W. A. van de Laar, J. P. Marrou-Mauriéres, A. P. J. Wijnands, H. J. Dane, S. G. Kruijswijk, and A. J. M. Houtsma, "A quasistationary model of air flow in the reed channel of single-reed woodwind instruments," Acustica 70, 146-154 (1990).

${ }^{11}$ C. J. Nederveen, Acoustical Aspects of Woodwind Instruments (F. Knuf, Amsterdam, 1969).

${ }^{12} \mathrm{~S}$. C. Thompson, "The effect of the reed resonance on woodwind tone production," J. Acoust. Soc. Am. 66, 1299-1307 (1979).

${ }^{13}$ S. K. Mitra, Digital Signal Processing. A Computer Based Approach (McGraw-Hill, New York, 1998).

${ }^{14} \mathrm{D}$. Rocchesso and F. Turra, "A generalized excitation for real-time sound synthesis by physical models," in Proc. Stockholm Mus. Acoust. Conf., Stockholm (1993), pp. 584-588.

${ }^{15}$ M. van Walstijn, "Discrete-Time Modelling of Brass and Reed Woodwind Instruments with Applications to Musical Sound Synthesis," Ph.D. dissertation, Dept. of Physics and Astronomy, University of Edinburgh, 2001.

${ }^{16}$ J. D. Lambert, Numerical Methods for Ordinary Differential Systems (Wiley, New York, 1993).

${ }^{17}$ C. Wan and A. M. Schneider, "Further improvements in digitizing continuous-time filters," IEEE Trans. Signal Process. 45(3), 533-542 (1997).

${ }^{18}$ A. M. Schneider, J. T. Kaneshige, and F. D. Groutage, "Higher order s-to-z mapping functions and their application in digitizing continuoustime filters," Proc. IEEE 79(11), 1661-1674 (1991).

${ }^{19}$ F. Avanzini, "Stable and Accurate Numerical Methods in Physical Models of Musical Instruments. A Study on the Clarinet," in Proceedings of the Diderot Forum on Mathematics and Music, Vienna (1999), pp. 11-19.

${ }^{20}$ D. H. Keefe, "On Sound Production in Reed-driven Wind Instruments," Systematic Musicology Technical Report 9003, School of Music, University of Washington, Seattle (1990).

${ }^{21}$ C. Maganza, R. Caussé, and L. Laloë, "Bifurcations, period doublings and chaos in clarinetlike systems," Europhys. Lett. 1(6), 295-302 (1986).

${ }^{22}$ E. D. Scheirer, "Structured audio and effects processing in the MPEG-4 multimedia standard," Multimedia Syst. 7(1), 11-22 (1999).

${ }^{23}$ G. P. Scavone, "An Acoustic Analysis of Single-Reed Woodwind Instruments with an Emphasis on Design and Performance Issues and Digital Waveguide Modeling Techniques," Ph.D. dissertation, Dept. of Music, Stanford University, 1997.

${ }^{24}$ See $\langle$ www.dei.unipd.it/ avanzini/jasa2002/〉. 